\title{
Impact of chest wall motion caused by respiration in adjuvant radiotherapy for postoperative breast cancer patients
}

\author{
C. Lowanichkiattikul', M. Dhanachai, C. Sitathanee, S. Khachonkham and P. Khaothong
}

*Correspondence: chairat.lowa@gmail.com Faculty of Medicine, Ramathibodi Hospital, Mahidol University, 270 Rama Road VI, Rachathevi, Bangkok 10400, Thailand

\begin{abstract}
To determine the chest wall movement of each patient during deep inspiratory breath hold (DIBH) and expiratory breath hold (EBH) in postoperative breast cancer patients. Postoperative breast cancer patients who underwent CT simulation for 3D radiotherapy treatment planning during December 2012 to November 2013 were included. Before scanning the radio-opaque wire was placed on the surface for breast and chest wall visualization on $\mathrm{CT}$ images, then the patient underwent three phases of $\mathrm{CT}$ scanning (free breathing, $\mathrm{DIBH}$, and $\mathrm{EBH}$, respectively). The distances of chest wall motion at five reference points were calculated using the treatment planning system. 38 breast cancer patients who underwent surgery were included. Median age was 48.5 (28-85) years. Median BMI was $23.4(16.6-38.3) \mathrm{kg} / \mathrm{m}^{2}$. Median lung volume was 3160.5 (1830.8-4754.0) $\mathrm{cm}^{3}$. Median Haller index was $2.43(1.92-3.56)$. Median chest wall movement was wider in anteroposterior (A-P, 4.2-5.4 mm) than superoinferior (S-I, 2.5-2.6 mm) and mediolateral $(M-L, 0.6-1.1 \mathrm{~mm})$ dimension in all five measured points. There was no significant effect of the type of surgery, BMl, lung volume, and the Haller index on the distances of chest wall movement. Additional margins of 7, 5, and $2 \mathrm{~mm}$ to the A-P, S-I, and M-L dimension should adequately cover the extreme chest wall movement in $95 \%$ of the patients. This study showed that the maximal movement of the chest wall during DIBH and EBH was greatest in the A-P axis followed by the S-I axis, while the $M-L$ axis was minimally affected by respiration.
\end{abstract}

Keywords: Breast cancer, Adjuvant radiotherapy, Respiration, Chest wall motion

\section{Background}

Breast cancer is the most common cancer in women (Attasara and Buasom 2011; Siegel et al. 2012). It is known that postoperative breast irradiation can improve local control in early-stage breast cancer (Fisher et al. 2002). According to the anatomy of the breast that lies on the anterior chest wall, intra-fraction movement of the clinical target volume (CTV) can occur due to respiration during treatment delivery. Large treatment margins added to cover the movement may subsequently cause a substantial volume of normal tissue exposed to radiation resulting in increased risk of treatment-related toxicity (Korreman et al. 2006).

It has been assumed that breast motion from breathing during standard whole breast RT does not significantly affect the dose distribution within the breast tissue. In one

\section{望 Springer}

(c) 2016 Lowanichkiattikul et al. This article is distributed under the terms of the Creative Commons Attribution 4.0 International License (http://creativecommons.org/licenses/by/4.0/), which permits unrestricted use, distribution, and reproduction in any medium, provided you give appropriate credit to the original author(s) and the source, provide a link to the Creative Commons license, and indicate if changes were made. 
study the baseline average movement during normal breathing was $<2 \mathrm{~mm}$ in all dimensions (Chopra et al. 2006). However, in the extreme phase of respiration like DIBH the distance of chest wall movement can be increased up to about $12.6 \mathrm{~mm}$ (Pedersen et al. 2004) According to the European Organization for Research and Treatment of Cancer (EORTC) survey of clinical practice in Europe, there was still variation in an additional treatment margin with the mean value of around $7.5 \mathrm{~mm}$ (Hans and Coen 2013).

Lung vital capacity has been shown to strongly correlate with the chest wall movement (Cala et al. 1996). Chest wall deformity also affects the chest wall motion in patients with pectus excavatum, with the motion decreasing markedly at the level of deformity (Redlinger et al. 2011). The severity of deformity could be graded by Haller index, which was the ratio of the chest wall width and depth. Increasing Haller index indicated more severe deformity.

This study was planned to evaluate the movement distances of breast or chest wall during DIBH and EBH and also to evaluate factors affecting chest wall movement during respiration.

\section{Methods}

\section{Patients}

Consecutive postoperative breast cancer patients, planned for CT simulation for adjuvant radiotherapy in Ramathibodi Hospital from December 2012 to November 2013, were included in the study. Prospective data collection was performed. All patients provided written informed consent, and the study was approved by the institutional ethics committee and funded by the Faculty of Medicine, Ramathibodi Hospital, Mahidol University.

\section{CT simulation}

The patients lied on the breast board and were instructed to hold their breath for $20 \mathrm{~s}$ for both DIBH and EBH. Patients were trained and scanned in the supine treatment position with the ipsilateral arm lifted above the head and the sternum in a horizontal position. Scanning included a standard free-breathing (FB) scan, followed by DIBH and EBH. The DIBH and EBH scans started immediately after the patients' chest stabilized at the maximum height and were finished within the 20-s period to maintain breathholding position of the patients. During scanning, the patients were visualized by the researcher to ensure that they were in the intended breath-holding position. If that was not the case then re-scanning was performed. The area for FB scan included the entire neck and chest wall, whereas for the DIBH and EBH it included only the breast plus $2 \mathrm{~cm}$ margin (Korreman et al. 2006; Korreman et al. 2005). The DIBH and EBH scan used the same table speed and slice thickness to cover the entire target area within one single breath-holding cycle. For this reason we decreased the milliampere-seconds ( $\mathrm{mA} \mathrm{s}$ ) to both shorten the scan time and to reduce radiation exposure. This caused the reduction of the image quality but it was still good enough to see the radio-opaque markers. Table 1 showed details of the imaging protocol.

Orthogonal room lasers were used to position the patient and to place ink marks with radio-opaque markers on the skin surface. These markers were used to verify that the patient did not shift relative to the angle board and the CT table between the scan series 
Table 1 Details of the imaging protocol

\begin{tabular}{llrllll}
\hline Series & Range & mA s & $\mathbf{K v}$ & $\begin{array}{l}\text { Table speed } \\
(\mathbf{m m} / \mathbf{s})\end{array}$ & $\begin{array}{l}\text { Duration } \\
(\mathbf{s})\end{array}$ & $\begin{array}{l}\text { Slice thickness } \\
(\mathbf{m m})\end{array}$ \\
\hline FB & Neck, chest & 250 & 120 & 5 & 60 & 2.5 \\
DIBH/EBH & Chest & 80 & 120 & 5 & 20 & 2.5 \\
\hline
\end{tabular}

(Pedersen et al. 2004; Lu et al. 2000). For postmastectomy patients the radio-opaque wires were placed by the anatomical landmark to outline the CTV, superiorly at the level just below the clavicular head, inferiorly at $2 \mathrm{~cm}$ below the infra-mammary fold, medially at midline, and laterally at mid-axillary line, respectively. In patients who underwent breast-conserving surgery (BCS), the radio-opaque wires were placed along the breast contour. When the patients were positioned to the center of the CTV, the points where laser intersected with the CTV border ( $\mathrm{S}$ for superior, I for inferior, $\mathrm{M}$ for medial, $\mathrm{L}$ for lateral, and A for anterior at the CTV center) were used as the reference points to measure the chest wall movement. Figure 1 showed how the wires were placed in both groups of patients and the reference points as mentioned.

\section{Measurement of chest wall movement}

Data was collected using the Eclipse ${ }^{\circledR}$ planning system (Varian Medical Systems, Inc, California, USA). The translational movement of the reference points (S, I, M, L, and A) was recorded in all three dimensions, namely $\mathrm{S}-\mathrm{I}, \mathrm{A}-\mathrm{P}$, and $\mathrm{M}-\mathrm{L}$. The maximal movement in each direction of the reference points was calculated from the differences between the markers position during DIBH and EBH. Also the movement of the reference points during DIBH and EBH was compared to the FB position.

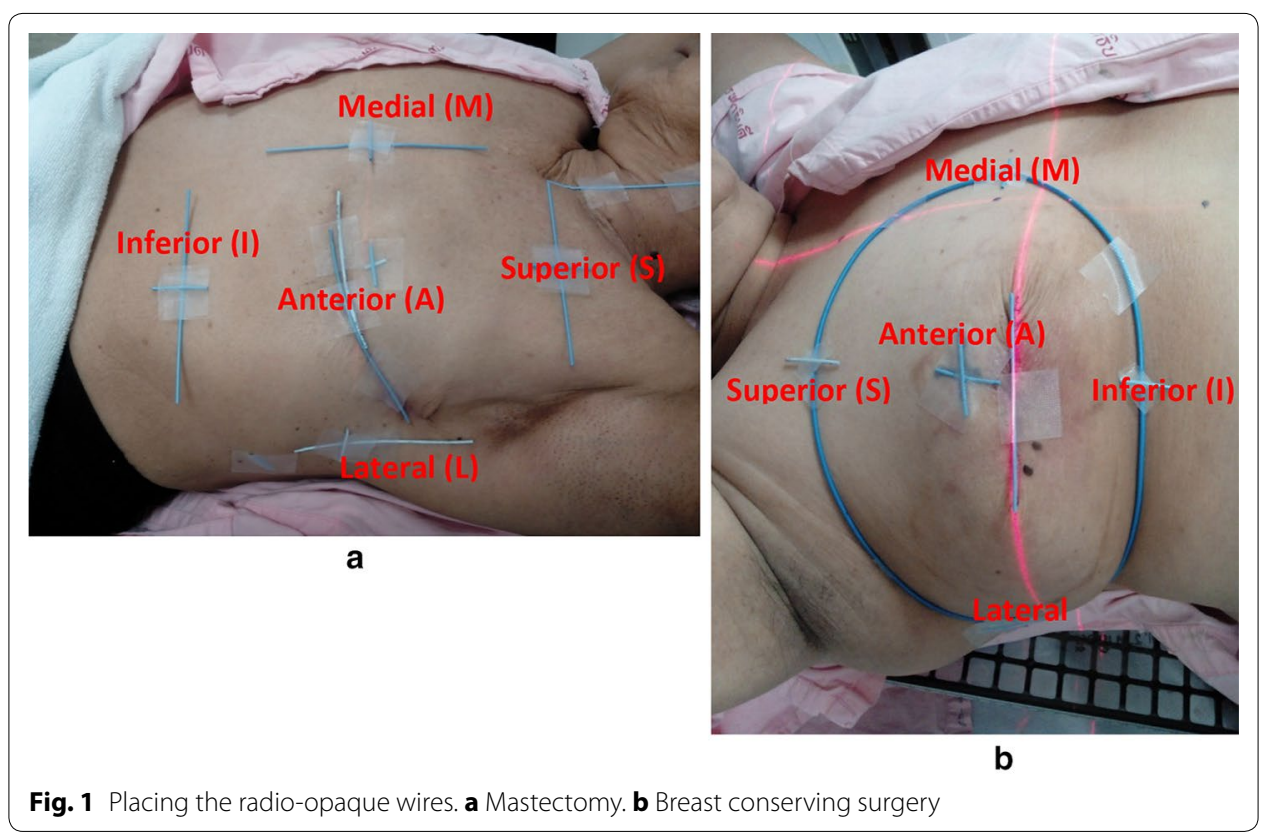




\section{Factors affecting chest wall movement}

Four factors were explored to find the correlation with chest wall movement including the surgical procedure (mastectomy vs BCS), the body mass index (BMI), the total lung volume during DIBH (lung volume $\mathrm{DIBH}_{\text {) }}$ ) and the Haller index.

BMI was calculated using the formula:

$$
B M I=\frac{\text { body weight }(\mathrm{kg})}{\text { height }^{2}\left(\mathrm{~m}^{2}\right)}
$$

Instead of the lung vital capacity, this study aimed to explore the correlation between chest wall movement and the total bilateral lung volume during DIBH. The volume was estimated using auto-contouring tools of the Eclipse ${ }^{\circledR}$ planning system.

The Haller index was used to grade the severity of chest wall deformity and was the ratio of the maximal width of chest wall divided by the minimal distance between the posterior sternum and anterior vertebral body at the same level. In this study, the index was measured in DIBH images (Redlinger et al. 2011).

\section{Statistical data analysis}

Mean, standard deviation (SD), median and range of the translational movement in each axis for each reference point was calculated. Spearman's rank correlation coefficient

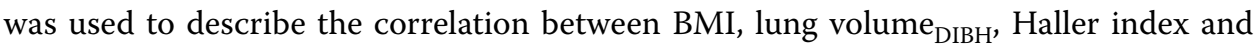
the chest wall movement. Mann-Whitney U test was used to compare the difference of chest wall movement between the mastectomy and BCS group.

\section{Results}

\section{Patients' characteristics}

38 patients were enrolled in the study, 21 had left-sided breast cancer. Median age was 48.5 (28-85) years. 27 patients underwent mastectomy and 11 had BCS. Median BMI was $23.4(16.6-38.3) \mathrm{kg} / \mathrm{m}^{2}$. Median lung volume ${ }_{\text {DIBH }}$ was $3160.5(1830.8-4754.0) \mathrm{cm}^{3}$. Median Haller index was 2.4 (1.9-3.6). The summary of the patients' characteristics was shown in Table 2.

\section{Chest wall movement}

Table 3 summarized the distances of the maximal chest wall movement in three axes of each marker. $95 \%$ coverage referred to the distance of chest wall movement of $95 \%$ of the patients. The median chest wall movement in the A-P axis was more pronounced than in the other axes in all five measured points, 4.2-5.4 mm. For the S-I axis the distance of movement was about $2.5 \mathrm{~mm}$. On the contrary the movement in the $\mathrm{M}-\mathrm{L}$ axis is less, $0.6-1.1 \mathrm{~mm}$. The margins that would cover the chest wall movement in $95 \%$ of the patients were $4.0-5.1 \mathrm{~mm}$ for the $\mathrm{S}-\mathrm{I}, 1.0-2.0 \mathrm{~mm}$ for the $\mathrm{M}-\mathrm{L}$, and $5.5-7.1 \mathrm{~mm}$ for the $\mathrm{A}-\mathrm{P}$ dimension.

Table 4 showed the distances of the chest wall movement during DIBH and EBH comparing to the chest wall position in the FB CT images. Movement in the posterior, superior and lateral direction was given the " + " sign. The distances of chest wall movement were greater during DIBH than EBH comparing to the FB scans in all dimensions 
Table 2 Patients' characteristics

\begin{tabular}{lcc}
\hline & Number (\%) & Median (range) \\
\hline Age (years) & $38(100)$ & $48.5(28-85)$ \\
BMI $\left(\mathrm{kg} / \mathrm{m}^{2}\right)$ & $38(100)$ & $23.4(16.6-38.3)$ \\
PS & & \\
ECOG 0 & $27(71.1)$ & \\
ECOG 1 & $11(28.9)$ & \\
Side & & \\
Left & $21(55.3)$ & \\
Right & $17(44.7)$ & \\
Staging & & \\
In situ & $7(18.4)$ & \\
I & $12(31.6)$ & \\
II & $9(23.7)$ & \\
III & $10(26.3)$ & $3160.5(1830.8-4754.0)$ \\
Procedure & & \\
Mastectomy & $11(28.9)$ & \\
BCS & $27(71.1)$ & \\
Haller's index & $38(100)$ & \\
Lung volume $\left(\mathrm{cm}^{3}\right)$ & $38(100)$ & \\
\hline
\end{tabular}

of almost all the measured points. During DIBH, the chest wall moved more anteriorly $(5.9-7.0 \mathrm{~mm})$ and superiorly $(2.5-5.0 \mathrm{~mm})$ than during FB. However, it moved $<1.0 \mathrm{~mm}$ in the $\mathrm{M}-\mathrm{L}$ dimension $(-0.7$ to $0.3 \mathrm{~mm}$ ). During $\mathrm{EBH}$ compared to $\mathrm{FB}$, the distances of movement were less. The chest wall moved 1.1-2.0 mm anteriorly, 0.0-1.2 mm superiorly and $0.1-0.3 \mathrm{~mm}$ mediolaterally.

\section{Factors affecting chest wall movement}

Different surgical procedure of the breast affected the shape of the chest wall, however this study showed no statistical significant differences in chest wall movement after mastectomy or BCS, except movement in S-I axis of the lateral marker, which showed more superior movement in BCS as $2.5 \mathrm{~mm}$ compared with $0.0 \mathrm{~mm}(\mathrm{p}=0.041)$.

For the other factors, data were analyzed to find the correlation between chest wall movement in each axis with BMI, lung volume ${ }_{\mathrm{DIBH}}$ and Haller index (HI). BMI and Haller index both showed no significant correlation with the chest wall movement. Comparing between normal BMI and obese patients, although the distances of chest wall movement in A-P direction of the patients with normal BMI were greater than in obese patients (4.7-6.5 mm compared to $2.6-4.3 \mathrm{~mm}$ ), the differences were not statistically significant. Movement in the other directions was almost the same in both groups of patients.

There was weak to moderate correlation between lung volume $\mathrm{DIBH}_{\text {and chest wall }}$ movement of only some measured points. In S-I axis, only I marker show significant moderate correlation with $\mathrm{r}_{\mathrm{s}}=0.405$. In A-P axis, I, L, and A marker had $\mathrm{r}_{\mathrm{s}}=0.390$, 0.486 and 0.361 , respectively. When lung volume ${ }_{\mathrm{DIBH}}$ was categorized into two groups, the patients with larger lung volume ${ }_{\mathrm{DIBH}}$ seemed to have more chest wall movement in A-P direction, 5.4-6.7 mm compared to $2.2-4.6 \mathrm{~mm}$ in patients with lung volume 
Table 3 The maximal chest wall movement

\begin{tabular}{|c|c|c|c|}
\hline & Mean & Median & $95 \%$ coverage \\
\hline & \multicolumn{3}{|c|}{ Point of intersection: superior (mm) } \\
\hline$S-1$ & $3.3 \pm 2.8$ & $2.5(0.0-10.0)$ & 4.2 \\
\hline$M-L$ & $1.5 \pm 1.5$ & $1.1(0.0-6.9)$ & 1.9 \\
\hline$A-P$ & $5.7 \pm 4.3$ & $5.4(0.0-20.0)$ & 7.1 \\
\hline & \multicolumn{3}{|c|}{ Point of intersection: inferior ( $\mathrm{mm}$ ) } \\
\hline$S-1$ & $3.0 \pm 2.9$ & $2.5(0.0-10.0)$ & 4.0 \\
\hline$M-L$ & $1.5 \pm 1.5$ & $1.1(0.0-5.8)$ & 2.0 \\
\hline$A-P$ & $5.8 \pm 4.0$ & $5.1(0.2-16.2)$ & 7.1 \\
\hline & \multicolumn{3}{|c|}{ Point of intersection: medial (mm) } \\
\hline$S-1$ & $3.9 \pm 3.4$ & $2.6(0.0-12.5)$ & 5.1 \\
\hline$M-L$ & $0.8 \pm 0.7$ & $0.6(0.0-3.5)$ & 1.0 \\
\hline$A-P$ & $5.3 \pm 3.8$ & $4.3(0.0-13.1)$ & 6.6 \\
\hline & \multicolumn{3}{|c|}{ Point of intersection: lateral (mm) } \\
\hline$S-1$ & $3.0 \pm 3.2$ & $2.5(0.0-12.5)$ & 4.0 \\
\hline$M-L$ & $1.2 \pm 1.4$ & $0.9(0.0-7.1)$ & 1.6 \\
\hline$A-P$ & $4.5 \pm 3.2$ & $4.2(0.0-12.0)$ & 5.5 \\
\hline & \multicolumn{3}{|c|}{ Point of intersection: anterior (mm) } \\
\hline$S-1$ & $3.6 \pm 2.6$ & $2.5(0.0-10.0)$ & 4.4 \\
\hline$M-L$ & $1.3 \pm 1.4$ & $1.0(0.0-7.7)$ & 1.7 \\
\hline$A-P$ & $5.7 \pm 4.2$ & $5.1(0.4-14.0)$ & 7.1 \\
\hline
\end{tabular}

Table 4 Distance of chest wall movement during DIBH and EBH comparing to the FB scan

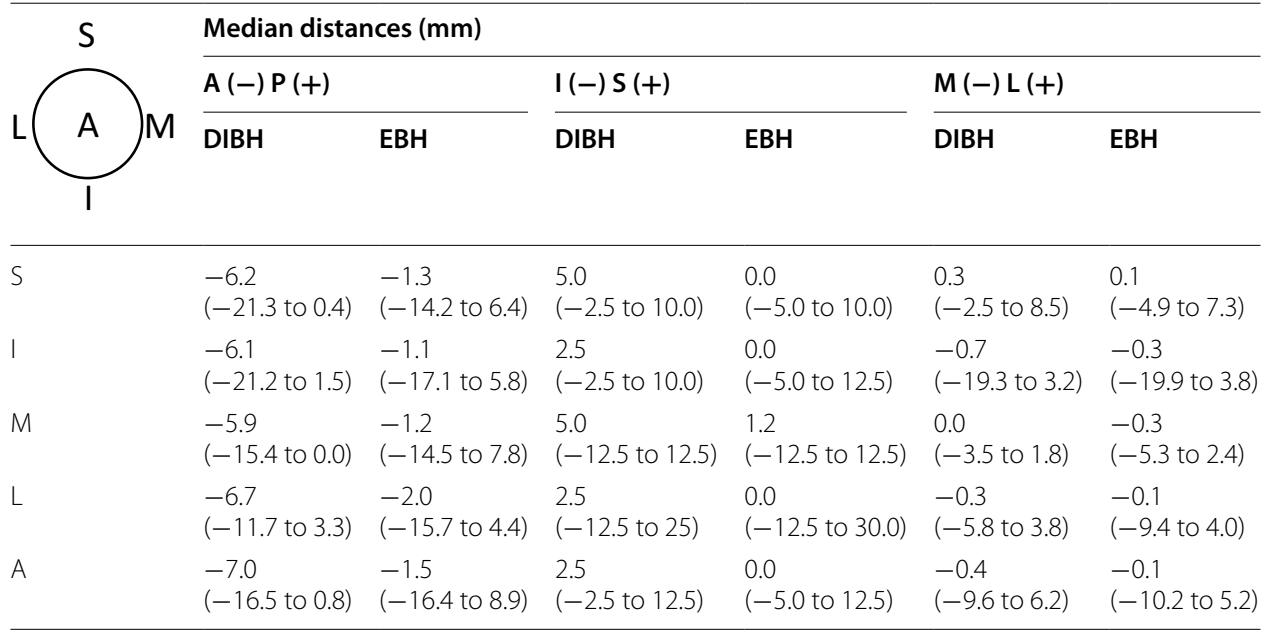


Table 5 Chest wall motion reported in different studies

\begin{tabular}{|c|c|c|c|c|c|}
\hline & \multirow[t]{2}{*}{$\mathbf{N}$} & \multirow[t]{2}{*}{ Method } & \multicolumn{3}{|l|}{ Result (mm) } \\
\hline & & & S-I & $M-L$ & $A-P$ \\
\hline Pedersen et al. (2004) & 16 & $\mathrm{DIBH}$ & - & - & $12.6(8.0-20.0)$ \\
\hline \multirow[t]{2}{*}{ Chopra et al. (2006) } & 5 & $\mathrm{DIBH}$ & $5.5(1.4-8.2)$ & $2.0(1.0-3.4)$ & $4.8(1.7-9)$ \\
\hline & & $\mathrm{FB}$ & $1.94(0.7-4.5)$ & $1.07(0.6-1.4)$ & $1.86(0.6-4.9)$ \\
\hline Kinoshita et al. (2008) & 17 & Tracking & $1.3 \pm 0.5$ & $1.0 \pm 0.6$ & $2.6 \pm 1.4$ \\
\hline \multirow[t]{2}{*}{ Richter et al. (2009) } & 10 & $4 \mathrm{DCT}$ & - & - & $1.8(0.2-3.8)$ \\
\hline & & EPID & & & $1.5(0.8-2.2)$ \\
\hline Wang et al. (2013) & 17 & $4 \mathrm{DCT}$ & $1.38 \pm 0.85$ & $1.03 \pm 0.48$ & $0.95 \pm 0.36$ \\
\hline This study & 38 & $\mathrm{DIBH}$ & & & \\
\hline Superior & & & $2.5(0.0-10.0)$ & $1.1(0.0-6.9)$ & $5.4(0.0-20.0)$ \\
\hline Inferior & & & $2.5(0.0-10.0)$ & $1.1(0.0-5.8)$ & $5.1(0.2-16.2)$ \\
\hline Medial & & & $2.6(0.0-12.5)$ & $0.6(0.0-3.5)$ & $4.3(0.0-13.1)$ \\
\hline Lateral & & & $2.5(0.0-12.5)$ & $0.9(0.0-7.1)$ & $4.2(0.0-12.0)$ \\
\hline Anterior & & & $2.5(0.0-10.0)$ & $1.0(0.0-7.7)$ & $5.1(0.4-14.0)$ \\
\hline
\end{tabular}

$\leq 3000$ cc. However, there were no statistical significant differences between the two groups in all other directions of chest wall movement.

\section{Discussion}

Many methods were used to evaluate the motion of the chest wall in breast cancer patients. This study showed how the respiration affected the chest wall movement by using the markers and conventional CT simulation. The comparison of chest wall movement reported from different studies is shown in Table 5. Our study confirmed that the movement in $\mathrm{A}-\mathrm{P}$ and $\mathrm{S}-\mathrm{I}$ direction was wider than in $\mathrm{M}-\mathrm{L}$ axis. The reason why overall chest wall movement in this study seemed to be larger than the previous studies using 4DCT or tracking technique might be the DIBH technique used to demonstrate the extreme phase of respiration. Comparing with the study reported by Chopra et al. which used the similar DIBH technique, the distance of chest wall movement seemed to be in the same magnitude except the movement in the S-I axis which was less in this study. This could be due to the position of the patients during CT scan using the breast board in the patient set up whereas in the previous study the chest wall movement was measured in the supine position without the breast board.

One reason that might explain why there was no strong correlation between different factors and the chest wall movement in this study was the relatively small number of patients with quite homogeneous baseline characteristics. Most patients were not obese with the BMI of $23.4 \mathrm{~kg} / \mathrm{m}^{2}$ and there was only one patient with the pectus excavatum. There seemed to be some relationship between the larger lung volume $\mathrm{DIBH}_{\mathrm{H}}$ and the more movement of the inferior, lateral, and the anterior measured points, which in some part might be explained by the more lung volume change in the lower lung region caused by the diaphragm reflecting in different movement of different part of the chest wall.

Naturally the chest wall moves unevenly during respiration, so applying the uniform margin for all directions in chest wall irradiation might result in over treating the normal tissue or under coverage of the CTV. Considering the distances of movement measured 
in different phases of breathing in this study the additional margins of 7, 5, and $2 \mathrm{~mm}$ to the A-P, S-I, and M-L dimension should adequately cover the extreme chest wall movement in $95 \%$ of the patients. Nevertheless, for clinical practice, margin adding should be individualized as much as possible. Also adding additional margins for setup error might be different from institute to institute.

\section{Conclusions}

This study show the maximal movement of the chest wall is in the anterior-posterior axis with is about $5.5-7.18 \mathrm{~mm}$ to coverage $95 \%$ of the patients so additional margin of $>7.1 \mathrm{~mm}$ especially in anterior-posterior axis is required to ensure the clinical target volume coverage from data of this study. But this study does not account for the error in interfraction patients' setup. So, the additional setup margin should be considered in each setting.

\section{Authors' contributions}

CL designed study, data collections, statistical analysis and drafted the manuscript. MD participated in the design of the study and revised the manuscript. CS participated in the design of the study. SK carried out the treatment planning. PK helped to design the computed tomography protocol. All authors read and approved the final manuscript.

\section{Acknowledgements}

This study was funded by Faculty of Medicine, Ramathibodi Hospital, Mahidol University, Thailand.

\section{Competing interests}

The authors declare that they have no competing interests.

Received: 7 December 2015 Accepted: 15 February 2016

Published online: 24 February 2016

\section{References}

Attasara P, Buasom B (2011) Hospital-based cancer registry, 26th edn. National Cancer Institute, Bangkok

Cala SJ, Kenyon CM, Ferrigno G, Carnevali P, Aliverti A, Pedotti A (1996) Chest wall and lung volume estimation by optical reflectance motion analysis. J Appl Physiol 81(6):2680-2689

Chopra S, Dinshaw KA, Kamble R, Sarin R (2006) Breast movement during normal and deep breathing, respiratory training and set up errors: implications for external beam partial breast irradiation. Br J Radiol 79:766-773

Fisher B, Anderson S, Bryant J, Margolese RG, Deutsch M, Fisher ER et al (2002) Twenty-year follow-up of a randomized trial comparing total mastectomy, lumpectomy, and lumpectomy plus irradiation for the treatment of invasive breast cancer. N Engl J Med 347(16):1233-1241

Hans P, Coen H. Breast radiotherapy technique: survey of practice in Europe. http://groups.eortc.be/radio/res/rtt_leeds/ s03_02hanspaulvanderlaan.pdf. Accessed 01 Dec 2013

Kinoshita R, Shimizu S, Taguchi H, Katoh N, Fujino M, Onimaru R et al (2008) Three-dimensional intrafractional motion of breast during tangential breast irradiation monitored with high-sampling frequency using a real-time tumortracking radiotherapy system. Int J Radiat Oncol Biol Phys 70(3):931-934

Korreman SS, Pedersen AN, Nottrup TJ, Specht L, Nystrom H (2005) Breathing adapted radiotherapy for breast cancer: comparison of free breathing gating with the breath-hold technique. Radiother Oncol 76(3):311-318

Korreman SS, Pedersen AN, Aarup LR, Nottrup TJ, Specht L, Nystrom H (2006) Reduction of cardiac and pulmonary complication probabilities after breathing adapted radiotherapy for breast cancer. Int J Radiat Oncol Biol Phys 65(5):1375-1380

Lu HM, Cash E, Chen MH, Chin L, Manning WJ, Harris J et al (2000) Reduction of cardiac volume in left-breast treatment fields by respiratory maneuvers: a CT study. Int J Radiat Oncol Biol Phys 47(4):895-904

Pedersen AN, Korreman S, Nystrom H, Specht L (2004) Breathing adapted radiotherapy of breast cancer: reduction of cardiac and pulmonary doses using voluntary inspiration breath-hold. Radiother Oncol 72(1):53-60

Redlinger RE, Kelly RE, Nuss D, Goretsky M, Kuhn MA, Sullivan Ket al (2011) Regional chest wall motion dysfunction in patients with pectus excavatum demonstrated via optoelectronic plethysmography. J Pediatr Surg 46:1172-1176

Richter A, Sweeney R, Baier K, Flentje M, Guckenberger M (2009) Effect of breathing motion in radiotherapy of breast cancer 4D dose calculation and motion tracking via EPID. Strahlenther Onkol 185(7):425-430

Siegel R, Naishadham D, Jemal A (2012) Cancer statistics, 2012. CA Cancer J Clin 62(1):10-29

Wang W, Li JB, Hu HG, Li FX, Xu M, Sun T et al (2013) Correlation between target motion and the dosimetric variance of breast and organ at risk during whole breast radiotherapy using 4DCT. Radiother Oncol 8:111 